\title{
Trap-assisted studies of odd, neutron-rich isotopes from Tc to Pd
}

\author{
J. Kurpeta • A. Jokinen • H. Penttilä • A. Płochocki • \\ J. Rissanen · W. Urban • J. Äystö
}

Published online: 4 April 2012

(C) The Author(s) 2012. This article is published with open access at Springerlink.com

\begin{abstract}
We review the present and future of trap-assisted structure studies of odd, neutron-rich $\mathrm{Tc}, \mathrm{Ru}, \mathrm{Rh}$ and $\mathrm{Pd}$ isotopes at the limits of present experimental techniques. These nuclei of refractory elements are produced in light-particle induced fission and filtered by their mass number with the IGISOL mass separator. Further mass separation with the JYFLTRAP Penning trap system provides a clean, monoisotopic beam perfectly suited for precise nuclear spectroscopy. Connecting the IGISOL and the JYFLTRAP facilities to the recently installed MCC30/15 cyclotron opens new prospects for post-trap spectroscopy of very exotic, neutron-rich nuclei.
\end{abstract}

Keywords Nuclear spectroscopy • Fission fragments • Mass separation • Ion trap

\section{Introduction}

For many years the IGISOL (Ion Guide Isotope Separator On-Line) technique [1] has been used to produce beams of neutron-rich, exotic nuclei from Tc to Pd. These elements are difficult to study due to their high melting points. With its stopping and

J. Kurpeta $(\bowtie) \cdot$ A. Płochocki · W. Urban

Faculty of Physics, University of Warsaw, ul. Hoża 69, 00-681 Warsaw, Poland

e-mail: jkurpeta@mimuw.edu.pl

\section{A. Jokinen · H. Penttilä · J. Rissanen · J. Äystö}

Department of Physics, University of Jyväskylä, P.O.Box. 35, 40014 Jyväskylä, Finland

W. Urban

Institut Laue-Langevin, 6 rue J. Horowitz, 38042 Grenoble, France 
transportation of fission products in a gas stream IGISOL provides ionized beams of $\mathrm{Tc}, \mathrm{Ru}, \mathrm{Rh}, \mathrm{Pd}$ and other refractory elements. A vast amount of data gathered at the front line of neutron-rich nuclei over past years with the IGISOL method is reviewed in this volume. A recent use of the JYFLTRAP tandem Penning trap system for producing isobarically purified ion beams considerably enhances the sensitivity and selectivity of nuclear spectroscopy studies.

The nuclei discussed here are created as light-particle-induced fission products. Typically beams of $20-30 \mathrm{MeV}$ protons or deuterons are used to induce fission process of natural uranium or thorium target. Fission products of interest are instantly transported by the IGISOL system to a dipole magnet which works as a mass separator. Its mass resolving power is suitable to separate nuclei of one mass number $A$ from all the other nuclear species produced in fission. These isobaric beams have successfully been used to study many exotic, neutron-rich nuclei of refractory elements, which were poorly known before the IGISOL technique. Many odd-mass, neutron-rich nuclei from Tc to $\mathrm{Pd}$ investigated for the first time with IGISOL were reported in [2].

After passing through a radio-frequency cooler and buncher [3], the isobaric beam from the IGISOL separator is injected into the purification trap of JYFLTRAP [4]. For fission products with $A=100$ mass resolving power of the trap reaches $10^{5}$ which is sufficient to separate a single isotope out of the isobaric beam. As a result, the Penning trap delivers a beam of one desired nuclide, here and after to be called a monoisotopic beam. For nuclear structure studies, the most interesting nuclei in each isobaric chain are the ones furthest away from the valley of beta stability. For the isobaric chains of neutron-rich technetium to palladium nuclei, the cross section usually drops by about one order of magnitude when going by one isobar away from stability. The more abundant, less exotic isobars result in many relatively strong lines in the $\gamma$ spectra. Their presence produce a lot of background events (mainly by Compton scattering) and sometimes they overlap with the usually weak lines of the exotic nuclei of interest. A huge background may cover weak lines of interest and overlapping background transitions make it hard to identify the peaks of interest as well as their intensities and half-lives. A monoisotopic beam of exotic nuclei is free from the dominating background of reaction-produced less exotic isobars. Thus, it is excellent for precise nuclear spectroscopy studies. For general review on JYFLTRAP, see [5] and a separate article in this special issue.

The combination of the ion-guide method and a Penning trap delivers monoisotopic samples of exotic nuclei which were not available before or were available only together with a huge amount of less interesting nuclides. Trap-assisted measurements investigate these very exotic nuclei with the well-known methods of $\gamma$ and $\beta$ coincidence spectroscopy providing reliable information on the nuclear structure.

\section{Recent achievements}

The neutron-rich nuclei from technetium to palladium $(43 \leq Z \leq 46)$ in the mass region $A \approx 110$ are located below the closed $Z=50$ proton shell and just above the $N=66$ neutron mid-shell. Nuclear shape in this area of the nuclide chart changes from strongly deformed in $\mathrm{Sr}$ and $\mathrm{Zr}$ around $N \geq 59$ to spherical closed shell at $Z=50$ in Sn isotopes. Shape coexistence phenomena, stable oblate deformation, 
Fig. 1 Systematics of the ground and low-energy excited states in odd, neutron-rich nuclei from Mo to $\mathrm{Pd}$. Monoisotopic beams of ${ }^{109,111}$ Mo, ${ }^{111,113}$ Tc and ${ }^{115} \mathrm{Ru}$ were produced using the combination of IGISOL and JYFLTRAP. The spin and parity assignments are tentative and brackets are omitted for better readability. The level energies are given in $\mathrm{keV}$, the $\mathrm{X}$ sign for ${ }^{113,115} \mathrm{Ru}$ indicates that the exact energy of the isomer is unknown. The data are based on refs. [2, 9-11, 15-29]. The estimation of the ${ }^{111} \mathrm{Mo}$ half-life is from [14]
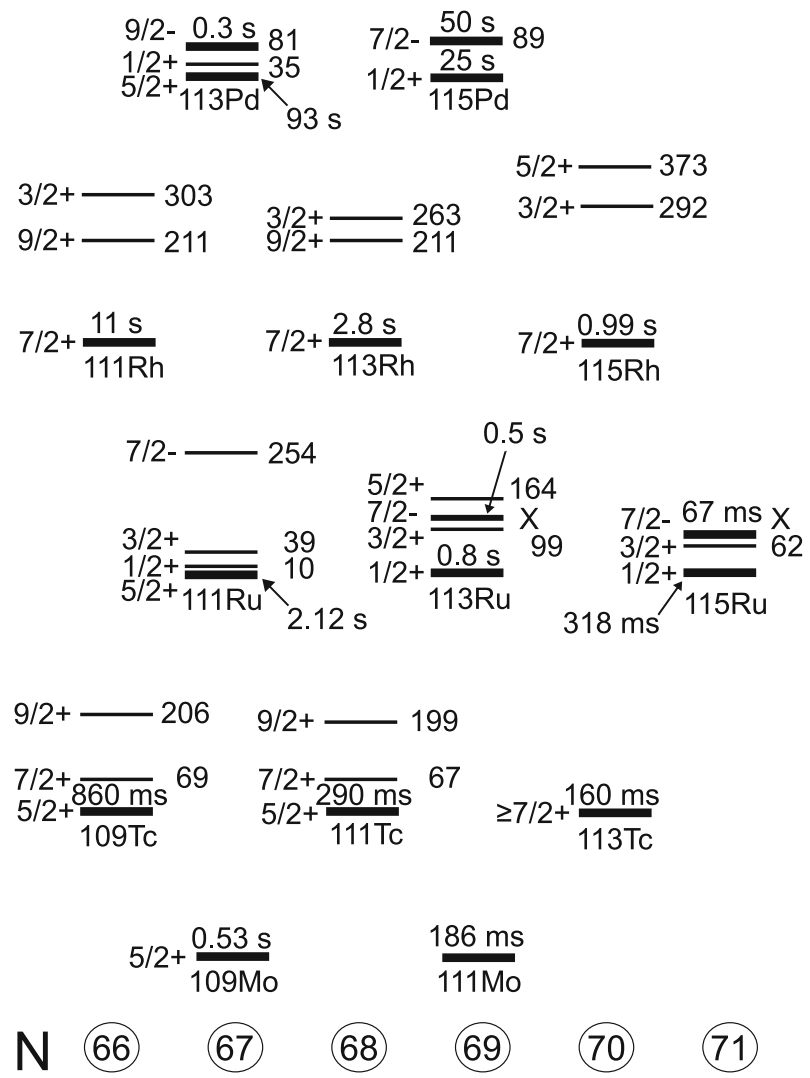

(71)

triaxial shapes and presence of isomeric states are the topics of current theoretical and experimental investigations in the region. Results of these studies are crucial for the development of nuclear structure models as well as for the modeling of the rapid neutron capture process ( $r$-process).

The JYFLTRAP Penning trap was used for nuclear spectroscopy for the first time in the $\beta$-decay experiment of even zirconium isotopes [6]. Soon after, the $\beta$-decay of ${ }^{115} \mathrm{Ru}$ monoisotopic samples [7] started the trap-assisted spectroscopy studies of Tc, $\mathrm{Ru}, \mathrm{Rh}$ and $\mathrm{Pd}$ isotopes. Further development of the isobaric beam purification in the Penning trap resulted in a preliminary extension of the ${ }^{115} \mathrm{Rh}$ level scheme fed by $\beta$ decay [8], discovery of an isomeric state in ${ }^{115} \mathrm{Ru}$ and finding a new half-life of its ground state $\beta$-decay [9], see Fig. 1 . It was also possible to study excited states in ${ }^{115} \mathrm{Pd}$, populated as a granddaughter in the $\beta^{-}$decay chain of the trap-purified ${ }^{115} \mathrm{Ru}$ [10]. Monoisotopic samples of ${ }^{113} \mathrm{Tc}$ were used to determine its $\beta$ decay half-life by observing the time decay pattern of the $98.5 \mathrm{keV} \gamma$ transition in ${ }^{113} \mathrm{Ru}$, see Fig. 2 . The experimental half-life value of $154(6) \mathrm{ms}$ is in accord with the previous results obtained with the IGISOL separator: 130(50) ms [11], 170(20) ms [12], and with inflight fragment separator $160_{-40}^{+50} \mathrm{~ms}$ [13]. Recent trap-assisted studies of ${ }^{109,111} \mathrm{Mo}$ $\beta$ decays provided new data on the structure of low-lying states in the daughter Tc isotopes, in particular signatures of oblate deformation in ${ }^{111} \mathrm{Tc}$ were found [14]. The results for ${ }^{109} \mathrm{Tc}$ will be published soon. 
Fig. 2 Decay of $98.5 \mathrm{keV}$ gamma line fed by $\beta^{-}$decay of

${ }^{113} \mathrm{Tc}$ monoisotopic samples

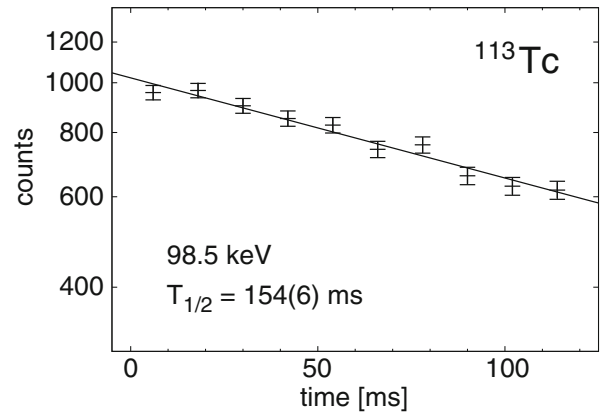

\section{Future of trap-assisted spectroscopy}

Our study of $\mathrm{Tc}, \mathrm{Ru}, \mathrm{Rh}$ and $\mathrm{Pd}$ isotopes is specifically motivated by the question of the structure of very neutron-rich isotopes in the region located close to the path of the astrophysical $r$-process. While the $r$-process nuclei are not yet accessible experimentally, the study of somewhat lighter isotopes may help to predict their properties, which are presently estimated only theoretically. For instance, the theory suggests that around the neutron number $N=70$, nuclei in this region develop an oblate shape, which becomes the ground-state configuration at $N>70$ [30]. On the other hand, the available experimental data for neutron-rich Pd isotopes are not in favor of such a picture. A recent theoretical work [31] on very neutron-rich $\mathrm{Zr}$ and Mo isotopes points to the important role of deformation and predicts almost equal potential minima for prolate and oblate shapes. Since the location of the $r$ process path depends on the nuclear deformation, it is now important to gather more experimental data in the critical region around $N=70$.

The theory [30] points to $\mathrm{Zr}$ isotopic chain as the one where the prolate-oblate transition should be the most pronounced. However, zirconium nuclei with $N \sim 70$ are presently unreachable for spectroscopy. The Tc, $\mathrm{Ru}$ and $\mathrm{Rh}$ isotopic chains are the nearest to $\mathrm{Zr}$, where one hopes to access the $N=70$ line in experimental spectroscopic studies. A possible way to verify the nuclear shape is to examine the single-particle structure of odd- $A$ and odd-odd nuclei. Such a structure in a prolatedeformed nuclear potential is different from that in an oblate one.

Below we present several ideas of improving and broadening the trap-assisted decay spectroscopy studies on the way towards more exotic nuclei located closer to the $r$-process path. The proposed enhancements are mainly based on the use of isobarically purified (so monoisotopic) ion beams in combination with various spectroscopic and mass separation methods. The monoisotopic beams are to be provided by the IGISOL mass separator coupled to JYFLTRAP system installed at the beam line of the new MCC30/15 cyclotron designed to deliver proton and deuteron beams with currents of 100 and $50 \mu A$, respectively.

Combining $\beta$ decay and prompt $\gamma$ data Detailed experimental investigation of nuclear structure is greatly improved when more than one experimental technique is available. The odd nuclei from technetium to palladium have been intensively studied in $\beta^{-}$decay experiments at IGISOL. Over the past decade a new tool has been developed, namely the measurements of multiple- $\gamma$ coincidences of prompt 
Fig. 3 Excited states in ${ }^{113} \mathrm{Ru}$ as an example of very different nuclear structures populated in the same nucleus by $\beta$ decay (left) and spontaneous fission (right). Data on ${ }^{113} \mathrm{Tc} \beta^{-}$ decay comes from IGISOL $[17,32]$ and on spontaneous fission of ${ }^{248} \mathrm{Cm}$ from prompt gamma-ray spectroscopy with the EUROGAM 2 array [21]

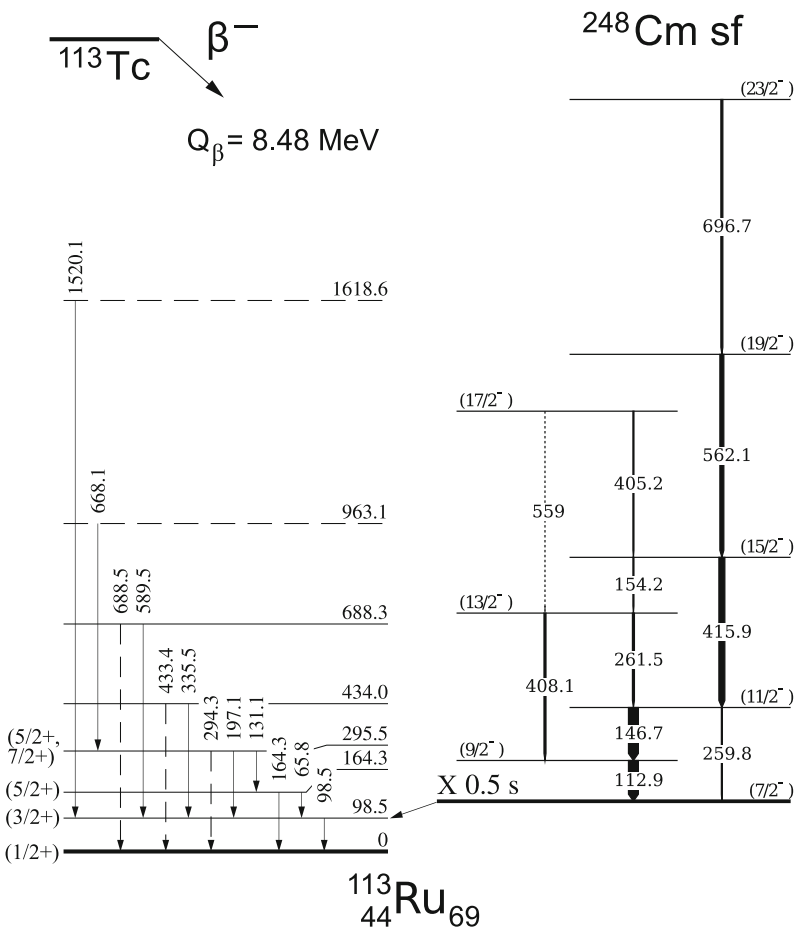

$\gamma$-rays following fission, collected using large arrays of germanium spectrometers. The structure of excited levels in a given nucleus fed by $\beta$-decay and spontaneous fission differs significantly. The spin values of the levels populated in $\beta$-decay are similar to the spin of the mother which is usually low. Sometimes a $\beta$-decaying isomer populates high-spin excited states, for example the $0.5 \mathrm{~s}$ isomer in ${ }^{113} \mathrm{Ru}$ [17]. High-energy levels populated in the daughter by $\beta$-decay depopulate either by many weak low-energy $\gamma$ transitions or a few high-energy ones. Both types are hardly detectable with germanium spectrometers due to their limited efficiency. Most often low-spin and low-energy nuclear levels are populated in the daughter nucleus via beta decay whereas spontaneous fission fragments are produced in high-spin, high-energy states which usually de-excite by long cascades of $\gamma$ transitions. The latter reveals band structures of nuclear excited levels. The $\beta$ decay data, among others, provides information on the band heads, especially their location relative to the ground state which is often hard to determine from prompt $\gamma$-ray studies. Furthermore, $\beta$ decay spectroscopy is sensitive to phenomena at the timescale of milliseconds, like some isomeric states, which cannot be measured by prompt $\gamma$ spectroscopy.

Combining both methods proved to be very successful as they provide complementary information while there is still some overlap for consistency checks. The IGISOL results on ${ }^{113} \mathrm{Tc}$ and ${ }^{113} \mathrm{Ru}$ decays [17, 32] with complementing ${ }^{248} \mathrm{Cm}$ prompt $\gamma$ spectroscopy data enabled to identify a new band in ${ }^{113} \mathrm{Ru}$ [21]. Thus, a revision of spins and parities for the states in ${ }^{113} \mathrm{Ru}$ and its daughter ${ }^{113} \mathrm{Rh}$ was possible, see Fig. 3. 
Half-life measurements with monoisotopic beams The half-life values of the exotic, neutron-rich nuclei are directly useful in the models of astrophysical $r$-process. The use of monoisotopic beam highly reduces background counts in the $\gamma$ spectra thus considerably improving the peak to background ratio. Such conditions permit halflives to be measured with a relatively low uncertainty as in the above mentioned example of ${ }^{113} \mathrm{Tc} \beta^{-}$decay (Fig. 2).

To determine the $\beta$-decay half-life one usually measures the half-lives of a few (or at least one) of the most intensive $\gamma$ lines in the daughter. Such decay measurements are usually done after a mass separation of the reaction products. A typical mass separator delivers a mixture of the desired nuclide and a few others which may be considered as background (although often more intensive than the wanted nuclide). In case of the IGISOL facility the background is formed by the less exotic isobars more easily produced in fission.

It may accidentally happen that the $\gamma$ line used for the half-life estimation contains an unwanted component (of a very similar energy unresolvable in a Ge spectrometer) emitted by one of the "background" isobars. As the half-lives of the unwanted isobars are always longer than of the most exotic one in the isobaric chain such a contamination makes the measured half-life longer. An illustrative example is the case of the ${ }^{115} \mathrm{Ru} \beta^{-}$decay half-life. For the first time, it was measured as 740(80) ms [11] with the isobaric beam from the IGISOL separator. Later, reinvestigation of the same decay with a monoisotopic beam from the trap, showed a shorter value of 318(19) ms [9]. The longer half-life in the first measurement was most probably caused by an overlapping $\gamma$ line emitted in the $\beta^{-}$decay of ${ }^{115} \mathrm{Pd}$, naturally present in the IGISOL isobaric beam.

When doing trap-assisted spectroscopy study, the daughter activities in the chain of consecutive $\beta$ decays are produced only by the decaying monoisotopic mother source. Therefore, any possible overlapping $\gamma$ lines coming from the isobars are of low intensity as compared to the mother activity delivered by the trap.

Moreover, with the trap one may always unambiguously distinguish the $\gamma$ lines fed by the most exotic $\beta$ decay from the ones fed by the less exotic isobars. By setting the trap to deliver a beam of the daughter of the activity of interest, disturbing $\gamma$-lines constituting the unwanted isobaric background in the gamma spectra are recorded. The method is also very useful for constructing the decay schemes.

Angular correlations and polarization of $\gamma$-rays In order to get relevant physical information one must complement the level schemes with information on transition multipolarities and mixing ratios. The multipole order of a gamma transition can be found by measuring its angular distribution. The simplest setup of three germanium detectors arranged at $90^{\circ}$ to each other, usually used for post-trap spectroscopy, can distinguish a dipole from a quadrupole transition. To achieve a higher resolution and sensitivity it is desirable to use a larger array of germanium detectors. In the case of odd nuclei it is especially important to use germanium detectors of high efficiency in the low-energy range, like those equipped with thin beryllium windows.

The experiments using the $\gamma-\gamma$ angular correlation technique were already successfully carried out at the IGISOL facility for palladium isotopes [33, 34]. This technique will benefit from the purity achieved with JYFLTRAP.

The angular distribution measurements cannot distinguish between the electric and magnetic multipoles which is possible via a determination of the linear 
polarization of the $\gamma$ radiation. Moreover, linear polarization measurements make it possible to unambiguously find multipole mixing ratios. The basic technique for finding polarization of $\gamma$-rays is Compton scattering. In odd nuclei one expects a high density of $\gamma$ lines at low energies. Thus, a good resolution in the low-energy range is an important parameter. An example of a germanium Compton polarimeter with a high resolution at low energies is described in [35]. It is a planar device with electrically defined sectors, compactly assembled in one cryostat and capable of completing the polarization measurements without changing its orientation.

Measurements of conversion electrons The $\beta$-decay process often populates excited states in the daughter nucleus which de-excite by $\gamma$ transitions or via the competing process of internal conversion. For a chosen nucleus, the internal conversion probability increases with the multipole order of the transition and decreases strongly with the energy (inversely proportional to the $3^{\text {rd }}$ or $4^{\text {th }}$ power). The internal conversion coefficient (ICC), defined as the probability of electron emission relative to $\gamma$ emission, carries information on the parity and multipole order of the transition.

Low-energy and high-multipolarity transitions are often associated with isomers. Some isomeric states in odd palladium and ruthenium isotopes are shown in Fig. 1. For highly-converted transitions, the ICC can be determined using only $\gamma$ spectrometers with the intensity balance and the fluorescence methods if there exists a $\gamma$ line in coincidence with the isomeric transition. Both methods depend on the details of the decay scheme and some other factors like coincidence efficiency. Additionally the fluorescence method depends on the observation of x-rays. For $\mathrm{Tc}, \mathrm{Ru}, \mathrm{Rh}$ and $\mathrm{Pd}$ nuclei these are in practice $K_{\alpha}$ x-rays of energies around $18-21 \mathrm{keV}$.

A more universal method for determining ICCs is to record an electron spectrum with a dedicated detector. To find the transition multipolarity one determines experimentally the intensity ratio of the $\mathrm{K}$ and $\mathrm{L}$ electron peaks, and $\gamma$ spectrum is not necessary. The traditional conversion electron spectroscopy can be considerably improved by measuring conversion electrons from monoisotopic samples inside a Penning trap. A radioactive source in a form of an ion cloud trapped by the magnetic field in vacuum does not suffer from interactions of emitted electrons with any surrounding material. The magnetic field of the trap transports the electrons to a detector placed far away from the source. The feasibility of such in-trap conversion electron spectroscopy has already been demonstrated at JYFLTRAP [36]. Among others, conversion electron spectra were measured for a short living, $T_{1 / 2}=19.1(7) \mathrm{ms}$, isomeric state in ${ }^{117} \mathrm{Pd}$ [36]. It is important to continue these intrap conversion electron studies to fix the multipolarities of the transitions in the exotic nuclei accessible at IGISOL.

Identification of isomeric transitions Isobarically purified ion samples are very useful for the identification of new isomeric transitions. The lack of contamination from unwanted reaction products and a high intensity of the nuclei of interest let us observe new isomeric transitions directly in the singles spectra. When using a germanium detector with a high efficiency at low energies one can observe both the isomeric $\gamma$ line and the conversion induced x-rays. By comparing the $\beta$-gated and the single $\gamma$ spectra one can immediately identify the isomeric $\gamma$ line as it is not present when a coincidence with a $\beta$ particle is required. As an example, the single and 
$\beta$-gated $\gamma$-ray spectra for the case of the $62 \mathrm{keV}$ isomeric transitions in ${ }^{115} \mathrm{Ru}$ are shown in Fig. 5 of [9].

Ground-state branching in $\beta$ decay There is no direct way to determine the ground state to ground state beta decay in a typical decay spectroscopy measurement using only $\gamma$ spectrometers and thin $\beta$ counters (for $\beta-\gamma$ coincidence). There is no $\gamma$-ray to be associated with such a transition and the energy cannot be determined with a transmission $\beta$ detector. Furthermore, it is difficult to reliably find the efficiency of such a detector at high $\beta$ energies typical for ground-state transitions of very exotic nuclei with $Q_{\beta}$ values close to $10 \mathrm{MeV}$. At JYFLTRAP the number of ions released from the trap can be counted with a movable microchannel plate (MCP) detector inserted into the beam. When the MCP is lifted up and the beam is implanted at the spectroscopy station the beam intensity is indirectly monitored by the number of $\gamma$ and $\beta$ events in the germanium and scintillation detectors, respectively. The fraction of the ions lost on the way from the MCP to the spectroscopy station is estimated by numerical simulations of ion trajectories. As the beam is purely monoisotopic, the total $\beta$-decay intensity can be measured with a combination of the MCP ion counts and the $\gamma$ and $\beta$ decay intensities measured at the spectroscopy station. Consequently, there is an experimental base to estimate the ground-state branching relevant for the determination of $\beta$ feedings and $\log f t$ values to the other populated levels.

Besides the above discussed ideas there are numerous ways to use the traps of the JYFLTRAP to complement and improve the quality of decay studies of refractory elements. The precision Penning trap is capable of determining nuclear masses and $Q_{\beta}$ values with a high accuracy. A new method of preparing isomerically clean samples of short-lived nuclei at JYFLTRAP [37] opens a field of trap-assisted spectroscopy of pure isomeric decays. Using a neutron detector after the trap for studying $\beta$-delayed neutron emission is of growing interest as the $Q_{\beta}$ values increase when going further from the stability. Last but not least, trap-assisted spectroscopy at IGISOL may offer new findings for the nuclides studied long time ago with less selective methods like chemical separation.

It is interesting to estimate the post-trap yields expected with the new MCC30/15 cyclotron providing proton beam currents up to $100 \mu A$ for the new IGISOL facility. Among the odd, neutron-rich Tc to Pd nuclei most of the trap-assisted measurements were done for the $A=115$ isobars. There, the last nuclide accessible for post-trap spectroscopy was ${ }^{115} \mathrm{Ru}$ and the yield for the more exotic ${ }^{115} \mathrm{Tc}$ was 2.1 ions/s for a $4 \mu \mathrm{A}$ proton beam and a $100 \mathrm{~ms}$ trap cycle. At higher cyclotron beam intensities, the IGISOL yield scales rather with the square root of the beam intensity [1]. Thus, increasing the proton beam current from 4 to $100 \mu A$ should increase the IGISOL yield by a factor of 5. Assuming that the Penning trap can fully accept the increased separator beam, a yield of 10.5 ions/s sufficient for post-trap spectroscopy studies of ${ }^{115} \mathrm{Tc}$ is expected. As the ${ }^{115} \mathrm{Tc}$ half-life is $73_{-22}^{+32} \mathrm{~ms}$ [38], using a trap cycle shorter than $100 \mathrm{~ms}$ would decrease the decay losses and provide higher yields. A decrease in the mass resolving power due to a shorter trap cycle is not so critical as the mass differences between neighboring isobars increase when going towards more exotic nuclei. Therefore, the most exotic isobars can be well separated even with a lower mass resolving power related to a shorter trap cycle. In conclusion, trap-assisted spectroscopy experiments at the new IGISOL facility may reach neutron-rich nuclei of interest located one isobar further away from the stability. 
Open Access This article is distributed under the terms of the Creative Commons Attribution License which permits any use, distribution, and reproduction in any medium, provided the original author(s) and the source are credited.

\section{References}

1. Äystö, J.: Nucl. Phys. A 693, 477 (2001)

2. Penttilä, H.: PhD Thesis, Dept. of Physics, University of Jyväskylä, Research Report No. 1/1992

3. Nieminen, A., Huikari, J., Jokinen, A., Äystö, J., Campbell, P., Cochrane, E.C.A., EXOTRAPS Collaboration: Nucl. Instrum. Methods A 469, 244 (2001)

4. Kolhinen, V., Eronen, T., Hager, U., Hakala, J., Jokinen, A., Kopecky, S., Rinta-Antila, S., Szerypo, J., Äystö, J.: Nucl. Instrum. Methods A 528, 776 (2004)

5. Jokinen, A., Eronen, T., Hager, U., Moore, I., Penttilä, H., Rinta-Antila, S., Äystö, J.: Int. J. Mass Spectrom. 251, 204 (2006)

6. Rinta-Antila, S., Eronen, T., Elomaa, V.-V., Hager, U., Hakala, J., Jokinen, A., Karvonen, P., Penttilä, H., Rissanen, J., Sonoda, T., Saastamoinen, A., Äystö, J.: Eur. Phys. J. A 311 (2007)

7. Kurpeta, J., Elomaa, V.-V., Eronen, T., Hakala, J., Jokinen, A., Karvonen, P., Moore, I., Penttilä, H., Płochocki, A., Rahaman, S., Rinta-Antila, S., Rissanen, J., Ronkainen, J., Saastamoinen, A., Sonoda, T., Urban, W., Weber, Ch., Äystö, J.: Eur. Phys. J A 31, 263 (2007)

8. Kurpeta, J., Rissanen, J., Elomaa, V.-V., Eronen, T., Hakala, J., Jokinen, A., Karvonen, P., Moore, I.D., Penttilä, H., Płochocki, A., Rahaman, S., Rinta-Antila, S., Ronkainen, J., Saastamoinen, A., Sonoda, T., Szerypo, J., Urban, W., Weber, Ch., Äystö, J.: Act. Phys. Pol. B 41, 469 (2010)

9. Kurpeta, J., Rissanen, J., Płochocki, A., Urban, W., Elomaa, V.-V., Eronen, T., Hakala, J., Jokinen, A., Kankainen, A., Karvonen, P., Małkiewicz, T., Moore, I.D., Penttilä, H., Saastamoinen, A., Simpson, G.S., Weber, C., Äystö, J.: Phys. Rev. C 82, 064318 (2010)

10. Kurpeta, J., Urban, W., Płochocki, A., Rissanen, J., Elomaa, V.-V., Eronen, T., Hakala, J., Jokinen, A., Kankainen, A., Karvonen, P., Moore, I.D., Penttilä, H., S. Rahaman, Saastamoinen, A., Sonoda, T., Szerypo, J., Weber, C., Äystö, J.: Phys. Rev. C 82, 027306 (2010)

11. Äystö, J., Astier, A., Enqvist, T., Eskola, K., Janas, Z., Jokinen, A., Kratz, K.-L., Leino, M., Penttilä, H., Pfeiffer, B., Żylicz, J.: Phys. Rev. Lett. 69, 1167 (1992)

12. Wang, J.C., Dendooven, P., Hannawald, M., Honkanen, A., Huhta, M., Jokinen, A., Kratz, K.-L., Lhersonneau, G., Oinonen, M., Penttilä, H., Peräjärvi, K., Pfeiffer, B., Äystö, J.: Phys. Lett. B 454, 1 (1999)

13. Pereira, J., Hennrich, S., Aprahamian, A., Arndt, O., Becerril, A., Elliot, T., Estrade, A., Galaviz, D., Kessler, R., Kratz, K.-L., Lorusso, G., Mantica, P.F., Matos, M., Möller, P., Montes, F., Pfeiffer, B., Schatz, H., Schertz, F., Schnorrenberger, L., Smith, E., Stolz, A., Quinn, M., Walters, W.B., Wöhr, A.: Phys. Rev. C 79, 035806 (2009)

14. Kurpeta, J., Urban, W., Płochocki, A., Rissanen, J., Pinston, J.A., Elomaa, V.-V., Eronen, T., Hakala, J., Jokinen, A., Kankainen, A., Karvonen, P., Moore, I.D., Penttilä, H., Saastamoinen, A., Weber, C., Äystö, J.: Phys. Rev. C 84, 044304 (2011)

15. Fong, D., Hwang, J.K., Ramayya, A.V., Hamilton, J.H., Luo, Y.X., Gore, P.M., Jones, E.F., Walters, W.B., Rasmussen, J.O., Stoyer, M.A., Zhu, S.J., Lee, I.Y., Macchiavelli, A.O., Wu, S.C., Daniel, A.V., Ter-Akopian, G.M., Oganessian, Yu.Ts., Cole, J.D., Donangelo, R., Ma, W.C.: Phys. Rev. C 72, 014315 (2005)

16. Penttilä, H., Enqvist, T., Jauho, P.P., Jokinen, A., Leino, M., Parmonen, J.M., Äystö, J., Eskola, K.: Nucl. Phys. A 561, 416 (1993)

17. Kurpeta, J., Lhersonneau, G., Płochocki, A., Wang, J.C., Dendooven, P., Honkanen, A., Huhta, M., Oinonen, M., Penttilä, H., Peräjärvi, K., Persson, J.R., Äystö, J.: Eur. Phys. J. A 13, 449 (2002)

18. Äystö, J., Taskinen, P., Yoshii, M., Honkanen, J., Jauho, P., Penttilä, H., Davids, C.N.: Phys. Lett. B201, 211 (1988)

19. Franz, G., Hermann, G., J. Inorg. Nucl. Chem. 40, 945 (1978)

20. Lhersonneau, G., Pfeiffer, B., Alstad, J., Dendooven, P., Eberhardt, K., Hankonen, S., Klöckl, I., Kratz, K.-L., Nähler, A., Malmbeck, R., Omtvedt, J.P., Penttilä, H., Schoedder, S., Skarnemark, G., Trautmann, N., Äystö, J.: Eur. Phys. J. A 1, 285 (1998) 
21. Kurpeta, J., Urban, W., Droste, Ch., Płochocki, A., Rohoziński, S.G., Rząca-Urban, T., Morek, T., Próchniak, L., Starosta, K., Äystö, J., Penttilä, H., Durell, J.L., Smith, A.G., Lhersonneau, G., Ahmad, I.: Eur. Phys. J. A 33, 307 (2007)

22. Pfeiffer, B., Lhersonneau, G., Dendooven, P., Honkanen, A., Huhta, M., Klöckl, I., Oinonen, M., Penttilä, H., Persson, J.R., Peräjärvi, K., Wang, J.C., Kratz, K.-L., Äystö, J.: Eur. Phys. J. A 2, 17 (1998)

23. Urban, W., Rząca-Urban, T., Droste, Ch., Rohoziński, S.G., Durell, J.L., Phillips, W.R., Smith, A.G., Varley, B.J., Schulz, N., Ahmad, I., Pinston, J.A.: Eur. Phys. J. A 22, 231 (2004)

24. Penttilä, H., Taskinen, P., Jauho, P., Koponen, V., Davids, C.N., Äystö, J.: Phys. Rev. C 38, 931 (1988)

25. Mehren, T., Pfeiffer, B., Schoedder, S., Kratz, K.-L.: Phys. Rev Lett. 77, 458 (1996)

26. Urban, W., Rząca-Urban, T., Durell, J.L., Smith, A.G., Ahmad, I.: Eur. Phys. J. A 24, 161 (2005)

27. Luo, Y.X., Hamilton, J.H., Rasmussen, J.O., Ramayya, A.V., Stefanescu, I., Hwang, J.K., Che, X.L., Zhu, S.J., Gore, P.M., Jones, E.F., Fong, D., Wu, S.C., Lee, I.Y., Ginter, T.N., Ma, W.C., Ter-Akopian, G.M., Daniel, A.V., Stoyer, M.A., Donangelo, R., Gelberg, A.: Phys. Rev. C 74, $024308(2006)$

28. Luo, Y.X., Rasmussen, J.O., Hamilton, J.H., Ramayya, A.V., Hwang, J.K., Zhu, S.J., Gore, P.M., Wu, S.C., Lee, I.Y., Fallon, P., Ginter, T.N., Ter-Akopian, G.M., Daniel, A.V., Stoyer, M.A., Donangelo, R., Gelberg, A.: Phys. Rev. C 70, 044310 (2004)

29. Urban, W., Droste, Ch., Rząca-Urban, T., Złomaniec, A., Durell, J.L., Smith, A.G., Varley, B.J., Ahmad, I.: Phys. Rev. C 73, 037302 (2006)

30. Xu, F.R., Walker, P.M., Wyss, R.: Phys. Rev. C 65, 021303 (2002)

31. Sarriguren, P., Pereira, J.: Phys. Rev. C 81, 064314 (2010)

32. Kurpeta, J., Lhersonneau, G., Wang, J.C., Dendooven, P., Honkanen, A., Huhta, M., Oinonen, M., Penttilä, H., Peräjärvi, K., Persson, J.R., Płochocki, A., Äystö, J.: Eur. Phys. J. A 2, 241 (1998)

33. Lhersonneau, G., Wang, J.C., Hankonen, S., Dendooven, P., Jones, P., Julin, R., Äystö, J.: Eur. Phys. J. A 2, 25 (1998)

34. Lhersonneau, G., Wang, J.C., Hankonen, S., Dendooven, P., Jones, P., Julin, R., Äystö, J.: Phys. Rev. C 60, 014315 (1999)

35. Sareen, R.A., Urban, W., Barnett, A.R., Varley, B.J.: Rev. Sci. Instrum. 66, 3653 (1995)

36. Rissanen, J., Elomaa, V.-V., Eronen, T., Hakala, J., Jokinen, A., Rahaman, S., Rinta-Antila, S., Äystö, J.: Eur. Phys. J. A 34, 113 (2007)

37. Eronen, T., Elomaa, V.-V., Hager, U., Hakala, J., Jokinen, A., Kankainen, A., Rahaman, S., Rissanen, J., Weber, C., Äystö, J.: Nucl. Instrum. Methods B 266, 4527 (2008)

38. Montes, F., Estrade, A., Hosmer, P.T., Liddick, S.N., Mantica, P.F., Morton, A.C., Mueller, W.F., Ouellette, M., Pellegrini, E., Santi, P., Schatz, H., Stolz, A., Tomlin, B.E., Arndt, O., Kratz, K.-L., Pfeiffer, B., Reeder, P., Walters, W.B., Aprahamian, A., Wöhr, A.: Phys. Rev. C 73, 035801 (2006) 